\title{
Flow study of an extracardiac connection with persistent left superior vena cava
}

\author{
Diane A. de Zélicourt, MSc, ${ }^{a}$ Kerem Pekkan, PhD, ${ }^{a}$ James Parks, MD, ${ }^{b}$ Kirk Kanter, MD, ${ }^{c}$ Mark Fogel, MD, \\ and Ajit P. Yoganathan, $\mathrm{PhD}^{\mathrm{a}}$
}

Supplemental material is available online.
From the Wallace H. Coulter Department of Biomedical Engineering, Georgia Institute of Technology and Emory University, Atlanta, Ga, ${ }^{a}$ Pediatric Cardiology, Emory University, Atlanta, Ga, ${ }^{\mathrm{b}}$ Department of Surgery, Emory University, Atlanta, Ga, ${ }^{c}$ and Division of Cardiology, The Children's Hospital of Philadelphia, Philadelphia, Pa. ${ }^{\mathrm{d}}$

This work was supported by a Bioengineering Research Partnership grant from the National Heart, Lung, and Blood Institute (HL67622). The glycerin used for the in vitro experiments was provided by $\mathrm{P} \& \mathrm{G}$ (Cincinnati, Ohio).

Received for publication Aug 25, 2005; revisions received Oct 17, 2005; accepted for publication Nov 8, 2005.

Address for reprints: Ajit P. Yoganathan, $\mathrm{PhD}$, Wallace H. Coulter School of Biomedical Engineering, Georgia Institute of Technology \& Emory University, Room 2119, U. A. Whitaker Building, 313 Ferst Dr, Atlanta, GA 30332-0535 (E-mail: ajit. yoganathan@bme.gatech.edu).

J Thorac Cardiovasc Surg 2006;131:785-91

$0022-5223 / \$ 32.00$

Copyright $(92006$ by The American Association for Thoracic Surgery

doi:10.1016/j.jtcvs.2005.11.031
Background: Numerous studies have sought to optimize the design of total cavopulmonary connections with a single superior vena cava. This study was directed to the $2 \%$ to $4.5 \%$ of the population with dual superior venae cavae, investigating the flow fields associated with such total cavopulmonary connection anatomies. Additionally, it demonstrates the potential use of computational designs and simulations as surgical planning tools.

Methods: A 3-dimensional model of a total cavopulmonary connection with bilateral superior venae cavae was reconstructed from a patient's magnetic resonance images and investigated experimentally and numerically to assess the power losses and flow structures within the connection. On the basis of these results, a virtual operation was performed in the computer to improve the original connection design. The modified anatomy was studied numerically.

Results: Because of a smooth connection with an extracardiac conduit and no major dimension mismatch between the baffle and the connecting vessels, the original anatomy yielded smooth flow fields, low power losses, and few disturbances. However, a large offset between the inferior vena cava and the left superior vena cava resulted in flow stasis and unbalanced hepatic flow distribution. Shifting the inferior vena cava and positioning it between the 2 superior venae cavae resulted in a $7 \%$ decrease in power losses and eliminated the associated flow stasis regions in the main pulmonary artery segment.

Conclusions: This study demonstrates the potential use of computer-aided design and numeric simulations for surgical planning. It shows that locating the inferior vena cava between the superior venae cavae may lead to better-balanced lung perfusion. This may require suturing the right and left superior venae cavae closer to each other during the hemi-Fontan or Glenn stage.

$\mathrm{D}$ espite marked improvements in surgical outcomes, Fontan patients still experience numerous and serious long-term complications. ${ }^{1}$ Among the multiple variables that determine the outcome and quality of life of these patients, one that allows for some degree of control is the surgically created design of the bypass connection. From a geometrical point of view, this problem is that of optimizing a double-inlet, double-outlet connection. Parametric in vitro and computational fluid dynamics (CFD) studies have attempted to identify the contribution of different geometric parameters to the overall flow fields. This has led to suggestions for improvement, such as including a caval offset ${ }^{2}$ or enlarging the inferior vena cava (IVC) anastomosis. ${ }^{3}$

These studies, however, do not account for patients with a persistent left superior vena cava (SVC). Normally, the left precardinal vein drains blood from the upper body into the primitive atrium during the first weeks of embryonic development and then joins its right counterpart, thus forming the SVC, and its distal part persists as 


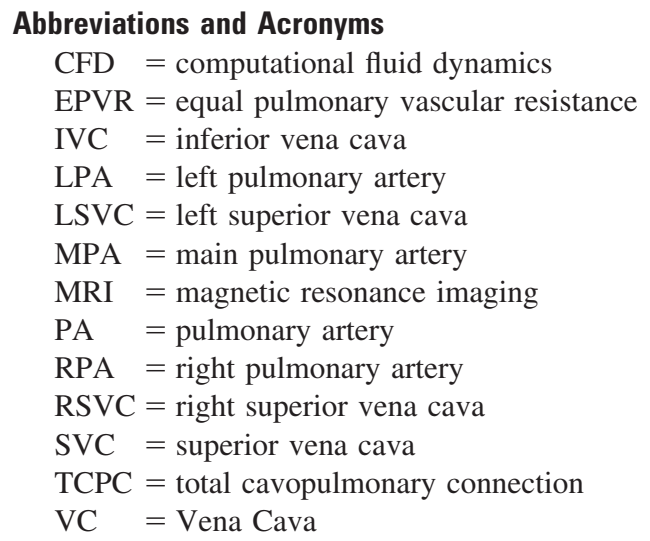

the coronary sinus. ${ }^{4}$ Persistent left SVC (LSVC) arises from a faulty embryogenesis between the fifth and eighth week and affects approximately $0.3 \%$ of the population. Its prevalence increases to $2 \%$ to $4.5 \%$ in patients with other congenital heart defects. ${ }^{5,6}$ The addition of that third inflow introduces supplementary parameters and issues into the quest for an optimal total cavopulmonary connection (TCPC) design and has not yet been investigated.

This study features an extracardiac TCPC with bilateral SVCs. The associated hemodynamic efficiency and flow structures were investigated by using a combined experimental and CFD approach. On the basis of these observations, an alternative surgical design is proposed and studied that demonstrates the potential applications of computeraided design and CFD tools to presurgical planning.

\section{Materials and Methods \\ Patient Data}

To investigate the variations of the TCPC anatomies and their effect on the TCPC flow structures and efficiency, a multicenter database of Fontan patient magnetic resonance images (MRIs) has been assembled. Informed consent was obtained, and all associated studies were approved by the institutional review boards of the Children's Hospital of Philadelphia, Children's Healthcare of Atlanta, the University of North Carolina, and the Georgia Institute of Technology.

The patient retained for this study was an 8-year-old white boy who was born with a hypoplastic left heart and persistent LSVC. He had undergone a bilateral cavopulmonary anastomosis at the age of 6 months, followed by an extracardiac TCPC at 24 months. Forty-five T1-weighted axial MRIs were acquired over the entire span of the connection with a $256 \times 192$-pixel field of view. In addition to the anatomical slices, magnetic resonance phase velocity maps were acquired, which provided 3-dimensional velocity information in the venae cavae: mean flow rates across the IVC, right SVC (RSVC), and LSVC were $1.1,0.5$, and $0.45 \mathrm{~L} / \mathrm{min}$, respectively.

Anatomical Model Reconstruction and Virtual Surgery The raw anatomical MRIs were first interpolated in the out-of-plane direction $^{7}$ to enhance the dataset resolution. The TCPC blood volume

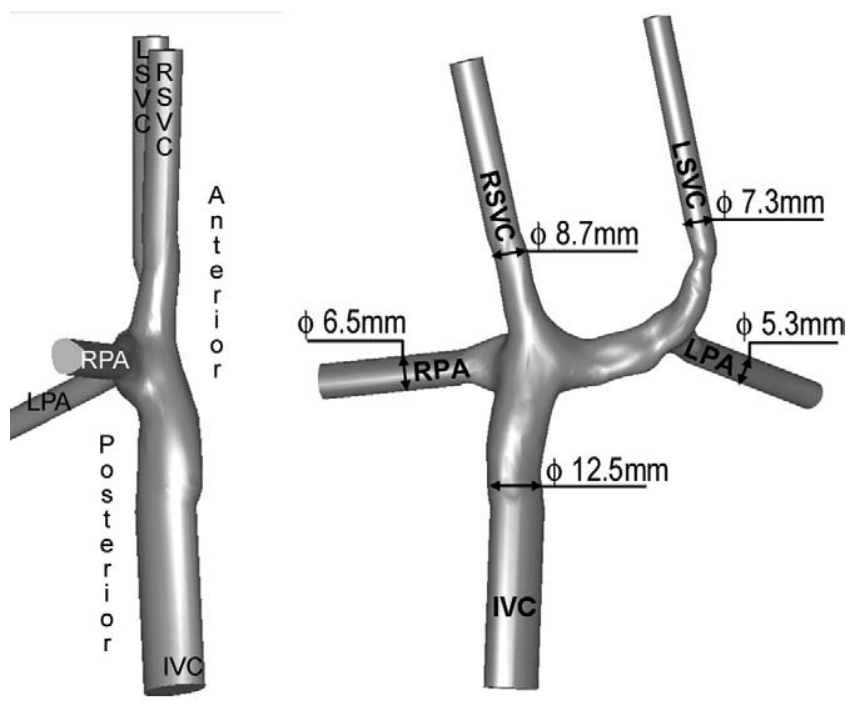

A. ORIGINAL ANATOMY

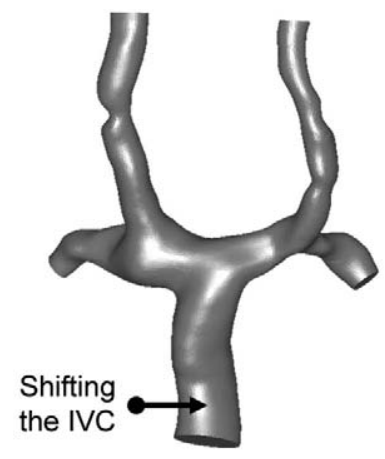

B. MODIFIED ANATOMY

Figure 1. A, Original TCPC configuration seen from a right and from an anterior perspective. B, Modified anatomy after the virtual operation was performed. The IVC was transected and displaced by $25 \mathrm{~mm}$ to lie in between the 2 SVCs.

(Figure 1, A) was reconstructed from the interpolated MRI data and used to generate both the numeric mesh and the experimental model. ${ }^{8}$

As will be discussed in the "Results" section, in vitro experiments and CFD results demonstrated significant flow stasis between the 2 SVCs. On the basis of this observation, an alternate surgical configuration was investigated. The IVC baffle was transected at the original IVC-to-RPA anastomosis site and shifted so as to lie in the middle of the 2 SVCs. The connection was smoothed out, thus yielding the morphology displayed in Figure 1, B. The virtual operation was performed by using a computer-aided design software package, GeomagicStudio 6.0 (Raindrop Geomagic, Research Triangle Park, NC).

Fluid Dynamic Analysis

The main criteria that were retained to characterize the TCPC hemodynamics were (1) the flow structure; (2) the power losses, 
which directly relate to the pressure drops across the connection; and (3) the distribution of the total venous return and of the IVC flow between the right (RPA) and left (LPA) pulmonary artery. A concise overview of the patient-specific, fluid dynamic analysis methodology is provided in the following paragraphs, and more detailed descriptions may be found in the studies by de Zélicourt and associates ${ }^{8}$ and Pekkan and colleagues. ${ }^{9}$

All experiments and CFD simulations were run under steady inflow conditions. Resting cardiac and caval flow ratios were taken from the flow data acquired in the scanner. Additional runs using the same caval flow ratios were performed at 3 and $4 \mathrm{~L} / \mathrm{min}$ to simulate active and exercise conditions.

Flow structure. The flow structures associated with this specific TCPC design were investigated both qualitatively and quantitatively by combining the experimental results from flow visualization and digital particle velocimetry with those of the numeric simulations.

Hydrodynamic power losses. The power losses across the connection were computed by using an integrated control volume energy balance ${ }^{2}$ :

$$
\dot{E}_{\text {Loss }}=\sum_{\text {Inlets }} P_{\mathrm{i}} \cdot Q_{\mathrm{i}}-\sum_{\text {Outlets }} P_{\mathrm{i}} \cdot Q_{\mathrm{i}},
$$

where $P_{\mathrm{i}}$ and $Q_{\mathrm{i}}$ are the static pressure (corrected for pressure head bias $^{10}$ in the experimental case) and the volumetric flow rate, respectively, in each vessel.

Equal pulmonary vascular resistance. To assess the quality of the lung perfusion associated with the original and the modified TCPC design, a simple lumped-parameter model was implemented to incorporate the pulmonary vascular resistance':

$$
\begin{gathered}
Q_{\mathrm{LPA}}+Q_{\mathrm{RPA}}=Q_{\mathrm{Total}} \\
R_{\text {Right }} \times\left(\frac{R_{\mathrm{Left}}}{R_{\text {Right }}} Q_{\mathrm{LPA}}-Q_{\mathrm{RPA}}\right)=P_{\mathrm{LPA}}-P_{\mathrm{RPA}},
\end{gathered}
$$

where $Q_{\mathrm{LPA}}, Q_{\mathrm{RPA}}, P_{\mathrm{LPA}}$, and $P_{\mathrm{RPA}}$ are the flow rates and static pressures in the LPA and RPA, and $R_{\text {Left }}$ and $R_{\text {Right }}$ the pulmonary vascular resistances of the left and right lungs. $P_{\mathrm{LPA}}$ and $P_{\mathrm{RPA}}$ are known from the experimental measurements or numeric simulations as a function of the flow split, $Q_{\mathrm{RPA}} / Q_{\mathrm{LPA}}$. In this study, both lung resistances were assumed to be equal and were set to an average pulmonary vascular resistance value of 1.8 Wood units, which provided us with the equal pulmonary vascular resistance (EPVR) operating point.

Note that $R_{\text {Left }}$ and $R_{\text {Right }}$ account for only the pulmonary resistances. The EPVR operating point depends on the resistance encountered by the flow when going from the Venae Cavae (VCs) to either one of the PAs. In a perfectly symmetric TCPC geometry, the pressure head needed to drive the flow into the LPA should equal the one needed to drive the flow into the RPA, thus resulting in an EPVR of 50:50 RPA/LPA.

\section{Results \\ Original Bilateral TCPC}

Anatomy. The patient anatomy featured in this study was characterized by (1) caval diameters of 12.5, 8.7, and $7.3 \mathrm{~mm}$ for the IVC, RSVC, and LSVC, respectively; (2) pulmonary diameters of 6.5 and $5.3 \mathrm{~mm}$ for the RPA and LPA, respec- tively; (3) an extracardiac conduit that yielded a smooth geometry for the IVC pathway; and (4) an IVC placed directly opposite the RSVC, whereas the LSVC was offset by $49 \mathrm{~mm}$.

Flow structure. Qualitative and quantitative assessments of the flow structures are shown in Figure 2. Run under steady flow conditions, the bilateral TCPC connection was characterized by smooth and steady flow fields. The only disturbances were observed at the point where the IVC and RSVC flow collided before going into the PAs. Because of the proximity of the LSVC to the LPA, the LSVC flow went exclusively to the LPA (Figure 2, $C$ and $G$ ). The intermediate PA section was perfused only by the blood coming from the IVC and RSVC and going into the LPA (Figure 2, A, B, E, F, I, and $J$; see also supplementary movies E1 and E2).

Varying the pulmonary flow splits affected only the IVC and RSVC flow fields and not the LSVC (supplementary movie E3). At 70:30 RPA/LPA, a recirculation region helped redirect the IVC and RSVC streams towards the RPA. Hardly any streaklines were observed in the intermediate main PA (MPA) segment, and digital particle velocimetry results revealed important flow stasis in this region. Because of the LSVC offset, the entire LSVC stream, which represented $22.5 \%$ of the flow, went directly into the LPA. Subsequently, at a 70:30 RPA/LPA flow split, only $7.5 \%$ of the flow traveled from the IVC/RSVC toward the LPA, thus resulting in regions of flow stasis in the MPA segment (Figure 2).

The perfusion of this intermediate MPA segment improved as more flow was directed to the LPA. At 30:70 RPA/LPA, the IVC and RSVC streams collided in the middle of the connection and splitted between the LPA and RPA. Most of the IVC and RSVC streams flowed along the inferior aspect of the MPA.

Pressure drops and power loss. No significant difference among the 3 caval pressures was demonstrated for all flow conditions $(P>.05)$. As expected, the pulmonary pressures decreased as more flow was forced through the corresponding PA. However, except at 70:30 RPA/LPA, the LPA pressure remained lower than that of the RPA for all the pulmonary flow splits, meaning that a higher IVC-to-PA pressure drop was needed to drive the flow through the LPA than through the RPA. Accordingly, the power losses calculated across the connection strongly depended on the RPA/LPA flow split, decreasing with an increasing RPA flow (Figure 3). As a result, this design favored right lung perfusion. The EPVR point corresponded to an RPA/LPA flow split of approximately 60:40 for all the tested cardiac outputs, going from 61:39 RPA/LPA at 2 and $3 \mathrm{~L} / \mathrm{min}$ to 62:38 RPA/LPA under exercise conditions at $4 \mathrm{~L} / \mathrm{min}$.

\section{Modified Anatomy}

Computing the EPVR point for the original TCPC demonstrated that with 2 lungs of equal resistance, this anatomy would tend to favor right lung perfusion. Additionally, flow 

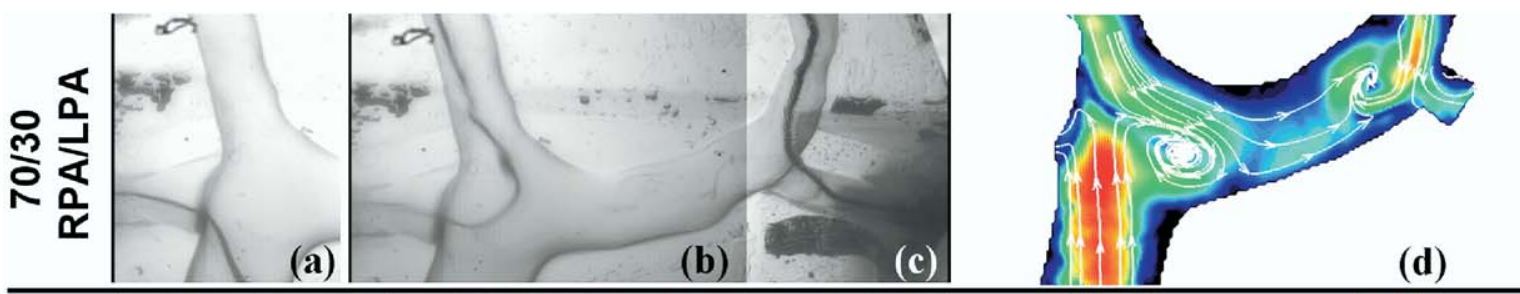

Velocity

(m/s)

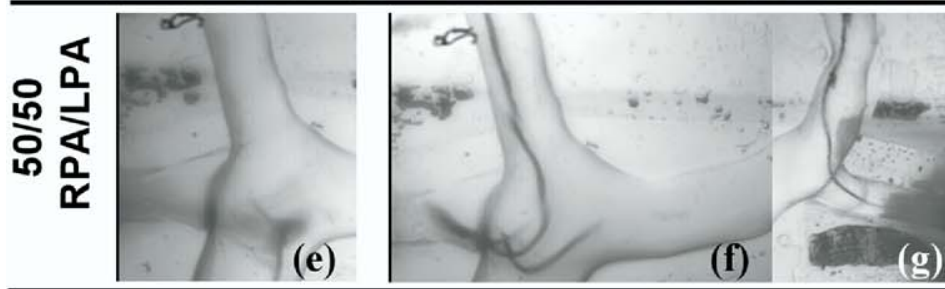

a)

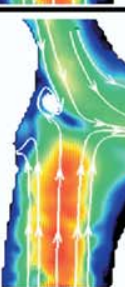

(d)
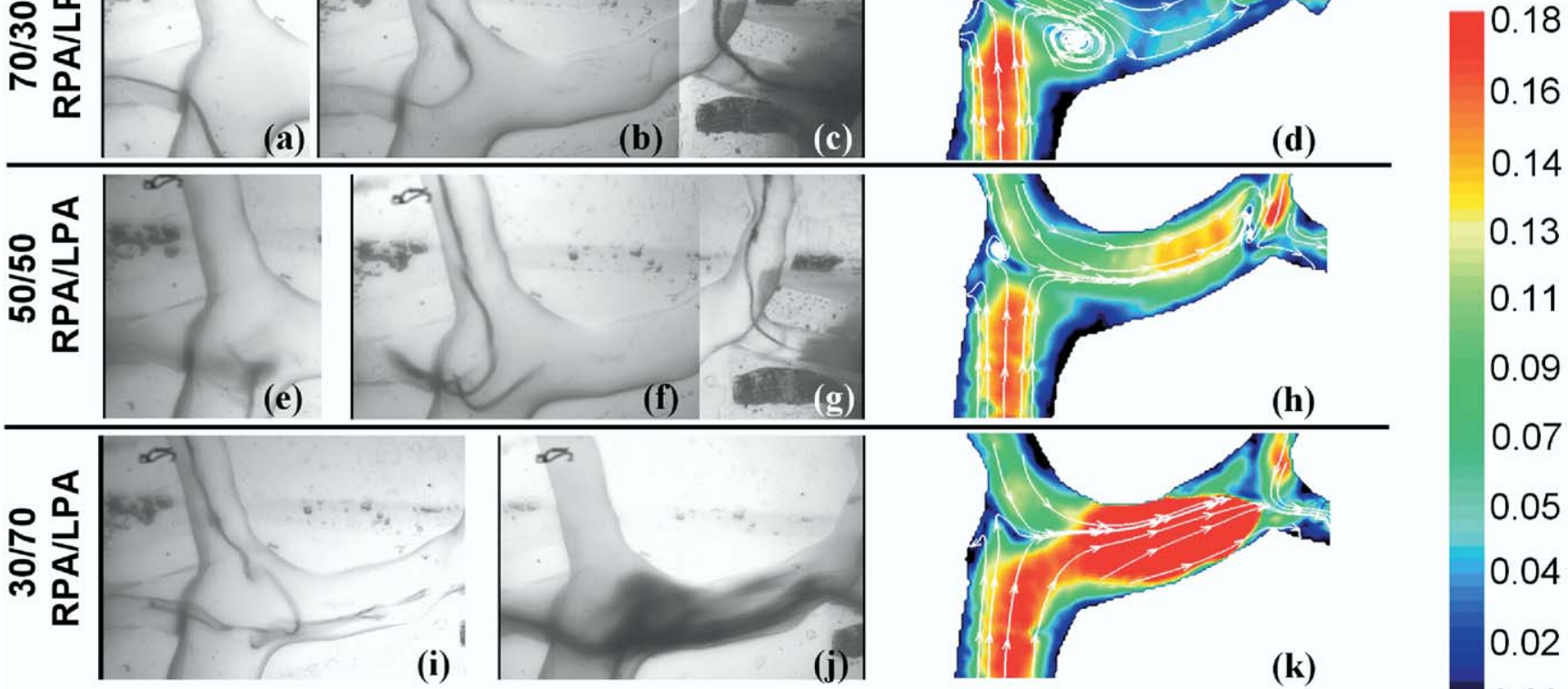

(h)

Figure 2. Qualitative (flow visualization) and quantitative (digital particle velocimetry) assessment of the flow structures observed in the original anatomy with a resting cardiac output of 2 L/min and varying RPA/LPA flow splits.

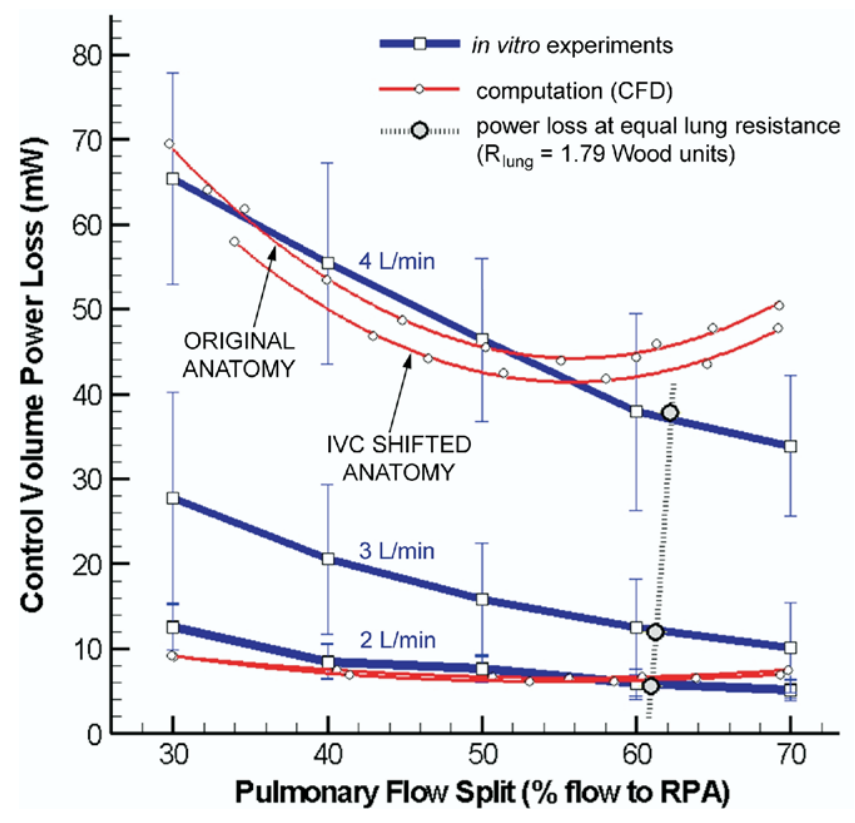

Figure 3. Experimental (b/ue) and numeric (red) power losses for the original and modified anatomies at total cardiac outputs of at 2, 3, and $4 \mathrm{~L} / \mathrm{min}$. The gray dashed line shows the flow split obtained in the original anatomy when an equal lung resistance of 1.8 Wood units was assumed. stasis was observed in the intermediate MPA segment when less than $50 \%$ of the flow went to the LPA. On the basis of these observations, a virtual operation was performed in an effort to reduce flow stasis and generate a more balanced flow field.

The power losses were $7 \%$ lower than in the original anatomy, and the EPVR did not significantly differ from that of the original anatomy. In the original configuration, at the EPVR point, most of the IVC flow went to the RPA (Figure 4, A-2). In the modified anatomy for the same flow split (Figure 4,B-2), the right and LSVCs flows went into the closest PA. The IVC stream splitted in 2 to adjust to the desired pulmonary flow split so that both lungs received a similar amount of hepatic flow. When compared with the original anatomy (Figure 4, A-1; see also the supplementary movie E4), this new configuration minimized the regions of flow stasis, and the only region with low flow velocities was the flow separation region at the IVC anastomosis site (Figure 4, $B-1$; see also the supplementary movie E5).

\section{Discussion}

The study presented in this article illustrated the flow fields associated with a typical bilateral SVC configuration and demonstrated the potential use of a combined computeraided design, CFD, and experimental approach in a clinical setting. 


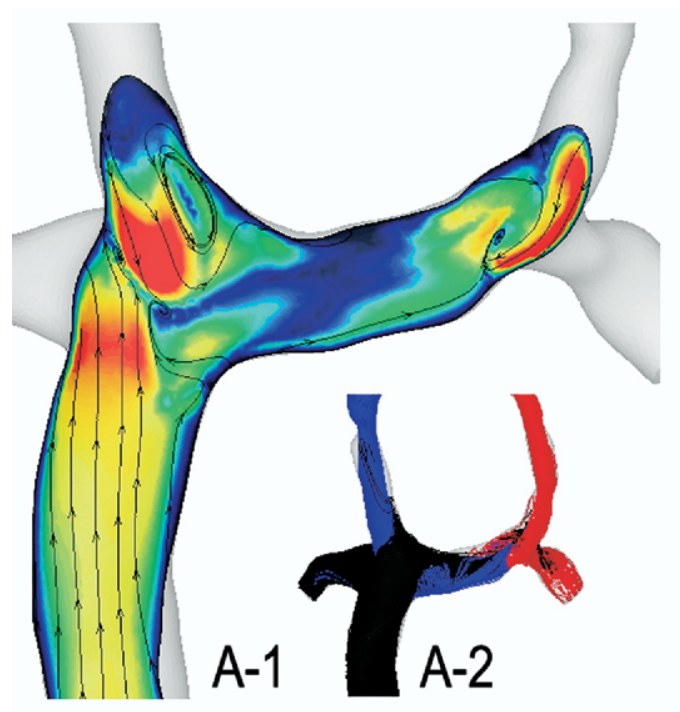

A. ORIGINAL ANATOMY
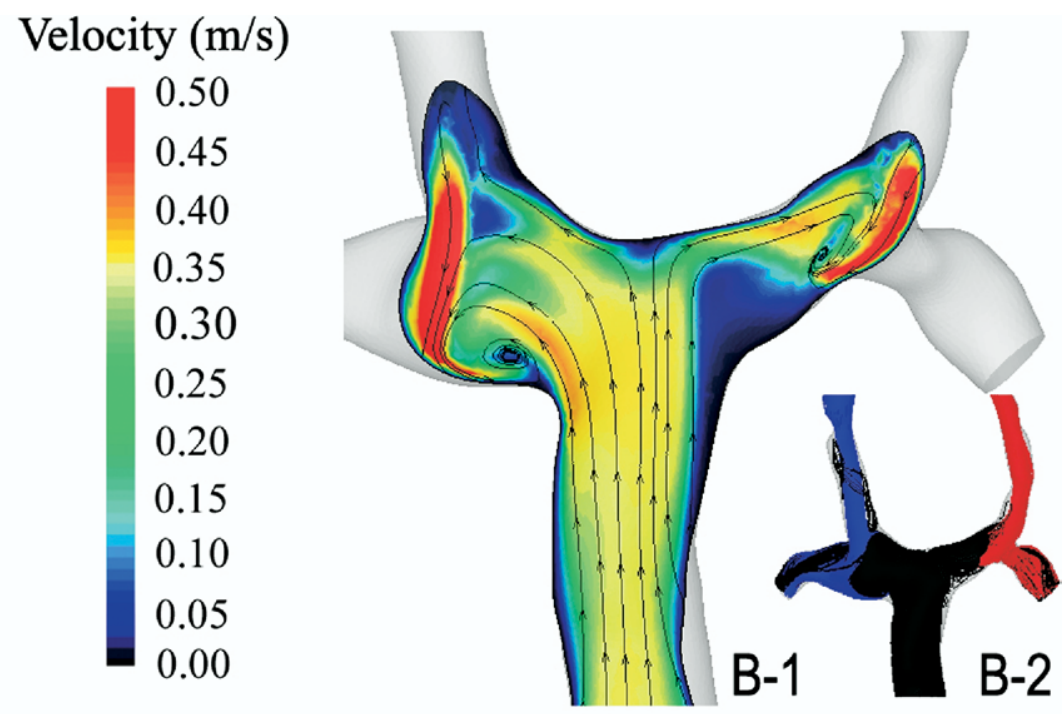

B. MODIFIED ANATOMY

Figure 4. CFD in-plane velocities (A-1 and B-1) and streamlines (A-2 and B-2) within the original (A) and modified (B) anatomies. Flow conditions included a total cardiac output of $4 \mathrm{~L} / \mathrm{min}$ and a pulmonary flow split of 65:35 RPA/LPA. The streamlines are color-coded in red for the IVC, blue for the right SVC, and red for the left SVC.

\section{PA Anastomosis Design and LPA Stenosis}

To provide some more insights into the effect of the different geometric parameters on the flow structures observed here, we compared our results with those of another study previously conducted in our laboratory on an intra-atrial TCPC with a single SVC. ${ }^{10}$ This former anatomy featured smaller vessels (hydraulic diameters of 13.1, 4.6, 5.4, and $4.6 \mathrm{~mm}$ for the IVC, SVC, RPA, and LPA, respectively) and an important mismatch between the baffle dimensions and the vessel diameters. This configuration generated important flow instabilities within the baffle and flow separation within the SVC. It also accelerated the flow as it went down the shrinking PAs, resulting in high pressure drops and power losses.

The PA diameters of this intra-atrial TCPC $2 \mathrm{~cm}$ downstream of the anastomosis site were of the same order as that of the bilateral SVC model, but the resulting pressure drops and power losses at the EPVR were 2 to 3 times lower in the bilateral SVC model than in the intra-atrial TCPC. This was believed to be due to the dimension of the connection area with respect to the connecting vessels and to the design of the PA anastomosis. In the bilateral SVC model, the extracardiac graft resulted in a smooth connection design. The PA diameters were only $30 \%$ smaller than the diameter of the corresponding anastomosis site, and the vessels were slowly tapered down to their final dimensions. In the intraatrial model, conversely, the tunnel resulted in a bulgy pouchlike connection design. The PA diameters were 50\% smaller than the corresponding anastomosis sites, and the vessels quickly tapered down to their final diameter. The fast tapering of the LPA accelerated the flow and reinforced the helical pattern.

These considerations are similar to assessing the effect of diffuse LPA hypoplasia on the TCPC energy consumption and lung perfusion. In their parametric study of LPA stenosis in Fontan patients, Pekkan and colleagues ${ }^{11}$ concluded that diffuse LPA hypoplasia of less than $40 \%$ was tolerable, whereas a larger one significantly decreased left lung perfusion and increased the energy consumption. As a general rule, changes in vessel diameters will result in energy losses. However, there is a threshold under which this increase will not be significant. For similar vessel dimensions, the dimensions of the connection area and the rate at which the PAs are tapered down to their final dimensions may result in drastically different flow and energy consumption patterns.

\section{Lung Perfusion}

The original connection design favors right lung perfusion, with an EPVR corresponding to a 60:40 RPA/LPA flow split. Lung perfusion is not perfectly balanced even in healthy subjects. However, having a large unbalanced flow perfusion is not desirable because it increases the overall energy dissipation and, thus, the workload imposed on the heart. 
To give a simple example, let us consider the energy dissipation through wall friction alone and assume both PAs to have equal diameters. Friction losses will be proportional to the square of the flow rate going through the corresponding vessel. If we have a perfectly balanced lung perfusion with $Q \mathrm{~L} / \mathrm{min}$ going through each $\mathrm{PA}$, the total power losses will scale as $2 Q^{2}$. In an unbalanced configuration with $(Q+$ $\Delta Q) \mathrm{L} / \mathrm{min}$ going to the RPA and $(Q-\Delta Q) \mathrm{L} / \mathrm{min}$ to the LPA, the power losses will scale as $2 Q^{2}+2 \Delta Q^{2}$. If the lung perfusion is only slightly unbalanced $(\Delta Q / Q \ll 1)$, then this increase will be negligible, but if the flow is truly unbalanced $(\Delta Q / Q$ no longer $\ll 1)$, the increase of $2 \Delta Q^{2}$ in overall losses will be significant. Large unbalanced lung flow may also affect the growth of the PAs, resulting in even more unbalanced lung perfusion and giving rise to the formation of collateral vessels.

\section{Hepatic Flow Distribution}

Another parameter of interest considered in this study was the hepatic flow distribution, which was assessed through the IVC flow distribution. In the original anatomy, the LSVC flow represented $22.5 \%$ of the total incoming flow and was entirely directed to the LPA. Subsequently, at the EPVR point only $17.5 \%$ of the flow went through the intermediate MPA section, resulting in regions of flow stasis. This implies that, in the original configuration, the IVC accounted for $22 \%$ and $81 \%$ of the LPA and RPA flows, respectively. This problem was not encountered in the modified anatomy, where the IVC was relocated in between the 2 SVCs and contributed in equal proportion to the LPA and RPA flows. Even if the exact contribution of hepatic flow to the development of the pulmonary vascular structure is still unclear, the exclusion of hepatic blood has been demonstrated to be strongly correlated with pulmonary venous malformations, ${ }^{12-14}$ and the contribution of the IVC flow to both lungs may be a criterion of importance.

\section{IVC Location}

Setting the IVC in between the 2 SVCs appeared as a good alternative: it decreased the power losses by $7 \%$ and had a significant effect on the lung perfusion, the distribution of the IVC flow, and the flow structures within the connection. Pekkan and colleagues ${ }^{15}$ developed a lumped parameter model of the Fontan circulation in which the TCPC was represented by the resistance it offered to the flow. When this model was applied to our study, the $7 \%$ decrease in power losses translated to a $7 \%$ decrease in TCPC resistance, which in turn led to a $10 \%$ increase in cardiac output under resting conditions.

In geometries with a single SVC, offsetting the IVC and SVC by 1.0 to 1.5 caval diameter minimizes the flow disturbances caused by the colliding inflows. ${ }^{2}$ Similarly, with dual SVCs, setting the IVC in between the 2 SVCs results in an offset between the IVC and each one of the SVCs, which in turn decreases flow instabilities, such as those observed in the original anatomy distal to the RPA (Figure 2,D).

Finally, setting the IVC in between the 2 SVCs drastically decreased the amount of flow stasis in the intermediate MPA segment. This is of major importance because vessels with little flow perfusion may not grow as well as other vessels and may be prone to thrombosis, which in turn will lead to additional complications. For example, considering the anatomy presented in this article, a smaller-diameter intermediate MPA segment would limit the perfusion of the left lung further and increase the vascular resistance.

In this case, our only manipulation was to shift the IVC by $24 \mathrm{~mm}$. However, the spatial constraints encountered by the surgeon may not allow for such a shift. Suturing the LSVC closer to the RSVC in the hemi-Fontan or Glenn stage may be a better way to decrease the distance by which the IVC should be shifted in the final TCPC stage.

\section{VC Anastomosis Type}

The connection studied here featured both a right hemiFontan and a left bidirectional Glenn. Looking at the shape of both connection types, the hemi-Fontan connection should have preferably directed the blood to one side, whereas the bidirectional Glenn should not have. However, in this bilateral SVC connection, the type of SVC anastomosis did not seem to have any real effect on the repartition of the SVC flows. Rather, it was the distance between the anastomosis sites (LSVC to LPA vs LSVC to RPA) that seemed to have a predominant effect. In the modified anatomy, the RSVC flow (with a hemi-Fontan connection) went exclusively to the RPA, whereas the LSVC flow (with a bidirectional Glenn) goes to the LPA. The IVC, positioned at equal distance from the 2 SVCs, splitted between the 2 PAs. The effect of the connection type may be more predominant in single-SVC cases, where the IVC and SVC should be close to each other.

\section{Toward Surgical Planning}

Figure 3 shows CFD and experimental results side by side. As has previously been pointed out by Pekkan and associates, ${ }^{9}$ the commercial CFD package used for these simulations captures the macro-scale, time-averaged features with good accuracy. Furthermore, the power losses are within 1 SD from the experimental results for RPA flow splits lower than $67 \%$. When $67 \%$ or more of the flow is directed to the RPA, the flow instabilities increase (Reynold's number = 2299 in the RPA at 70:30 RPA/LPA), reaching the limitations of our commercial CFD package.

In this study we used CFD and computer-aided design in combination to perform a virtual operation and assess its effect on the TCPC efficiency. This demonstrates the po- 
tential use of computer-aided design and CFD tools in surgical planning. However, as pointed out in the previous paragraph, the results should be considered with a critical mindset and weighed against the surgeon's judgment. Numerical simulations constitute a formidable tool, but their limitations should not be forgotten.

\section{Surgical Constraints}

It is important to keep in mind that this study only suggests some possible TCPC design improvements. In extracardiac cases, for example, shifting the IVC toward the LSVC may compress the pulmonary veins and worsen the patient's outcome rather than improving it. All the recommendations made in this article should thus be carefully balanced against the space constraints imposed by other vessels and organs and any other clinical considerations.

\section{Conclusions}

The original bilateral anatomy demonstrated smooth flow fields and low pressure drops and power losses. We believe that this was due to its uniform geometry, including the constant diameter of the artificial graft, and the smooth vessel connections, with only a small-dimensional mismatch between the baffle and the connecting vessels. Because of the position of the IVC, the connection design favored right lung perfusion. Shifting the IVC in between the 2 SVCs seemed to be a feasible and efficient way to obtain a better repartition of the hepatic flow to the lungs.

Using computer-aided design and CFD tools in combination, this study demonstrates potential developments in pediatric cardiac surgical planning. However, special attention should be paid to the strengths and limitations of the CFD modeling before any final clinical conclusions are drawn.

\section{References}

1. Gersony DR, Gersony WM. Management of the postoperative Fontan patient. Prog Pediatr Cardiol. 2003;17:73-9.
2. Sharma S, Goudy S, Walker P, Panchal S, Ensley A, Kanter K, et al. In vitro flow experiments for determination of optimal geometry of total cavopulmonary connection for surgical repair of children with functional single ventricle. J Am Coll Cardiol. 1996;27:1264-9.

3. de Leval MR, Dubini G, Migliavacca F, Jalali H, Camporini G, Redington A, et al. Use of computational fluid dynamics in the design of surgical procedures: application to the study of competitive flows in cavo-pulmonary connections. J Thorac Cardiovasc Surg. 1996;111: 502-13.

4. Hardey DW, White MS, Malloy KP, Hackett JE, Reed CC. Persistent left superior vena cava: review of embryologic anatomy and considerations for cardiopulmonary bypass. Cardiovasc Dis. 1980;7:433-41.

5. Campbell M, Deuchar DC. The left-sided superior vena cava. $\mathrm{Br}$ Heart J. 1954;16:423-39.

6. de Leval MR, Ritter DG, McGoon DC, Danielson GK. Anomalous systemic venous connection. Surgical considerations. Mayo Clin Proc. 1975;50:599-610.

7. Frakes DH, Conrad CP, Healy TM, Monaco JW, Fogel M, Sharma S, et al. Application of an adaptive control grid interpolation technique to morphological vascular reconstruction. IEEE Trans Biomed Eng. 2003;50:197-206.

8. de Zélicourt D, Pekkan K, Kitajima H, Frakes D, Yoganathan AP. Single-step stereolithography of complex anatomical models for optical flow measurements. J Biomech Eng. 2005;127:204-7.

9. Pekkan K, de Zélicourt D, Ge L, Sotiropoulos F, Frakes D, Fogel MA, et al. Physics-driven CFD modeling of complex anatomical cardiovascular flows-a TCPC case study. Ann Biomed Eng. 2005;33:284-300.

10. de Zélicourt D, Pekkan K, Wills L, Kanter K, Forbess J, Sharma S, et al. In vitro analysis of a patient-specific intraatrial total cavopulmonary connection. Ann Thorac Surg. 2005;79:2094-102.

11. Pekkan K, Kitajima H, de Zélicourt D, Forbess J, Parks J, Fogel $\mathrm{MA}$, et al. Total cavopulmonary connection flow with functional left pulmonary artery stenosis-angioplasty and fenestration in vitro. Circulation. 2005;22:3264-71.

12. Justino H, Benson LN, Freedom RM. Development of unilateral pulmonary arteriovenous malformations due to unequal distribution of hepatic venous flow. Circulation. 2001;103:E39-40.

13. Srivastava D, Preminger T, Lock JE, Mandell V, Keane JF, Mayer JE $\mathrm{Jr}$, et al. Hepatic venous blood and the development of pulmonary arteriovenous malformations in congenital heart disease. Circulation. 1995;92:1217-22.

14. Pike NA, Vricella LA, Feinstein JA, Black MD, Reitz BA. Regression of severe pulmonary arteriovenous malformations after Fontan revision and "hepatic factor" rerouting. Ann Thorac Surg. 2004;78:697-9.

15. Pekkan K, Frakes D, de Zelicourt D, Lucas CW, Parks J, Yoganathan AP. Coupling pediatric ventricle assist devices to the Fontan circulation: simulations with a lumped-parameter model. ASAIO J. 2005;51: 618-28. 